\title{
REVIEW
}

\section{Erector Spinae Block. A narrative review.}

\author{
María Bermúdez López, Álvaro Gasalla Cadórniga, José Manuel López González, \\ Enrique Domínguez Suárez, Carlos López Carballo, Francisco Pardo Sobrino \\ Anaesthesia and Pain management Department. Hospital Universitario Lucus Augusti, Lugo, \\ Galicia, Spain.
}

\section{Correspondence to:}

María Bermúdez López, Anaesthesia and Pain management Department. Hospital Universitario Lucus Augusti, Lugo, Galicia, Spain.

E-mail: majobl79@gmail.com

\section{Conflicts of interests}

Nothing to declare

\section{Acknowledgements \\ None}

Keywords: erector spinae block; anatomy; mechanism of action; indications.

These authors take responsibility for all aspects of the reliability and freedom from bias of the data presented and their discussed interpretation.

Central Eur J Clin Res 2018;1(1):28-39

Received: 11.07.2018, Accepted: 1.09.2018, Published: 05.09.2018

Copyright @ 2018 Central European Journal of Clinical Research. This is an open-access article distributed under the Creative Commons Attribution License, which permits unrestricted use, distribution, and reproduction in any medium, provided the original work is properly cited.

\section{Abstract}

The erector spinae plane block is a novel ultrasound-guided technique that has recently been described for the management of acute and chronic thoracic pain. Currently an increasingly number of indications for the ESP block have been published. Nevertheless, the anatomy, mechanism of action, doses and volume of local anesthetic needed are still unclear. The aim of this narrative review is study this new block with base on the updated medical literature.

\section{Introduction}

The erector spinae plane block (ESP block) is a novel ultrasound-guided technique that has recently been described for the man- agement of acute and chronic thoracic pain. ESP block is a regional anesthesia technique in which local anesthetic (LA) is injected between the erector spinae muscle and transverse process under ultrasound guidance, blocking the dorsal and ventral rami of the thoracic and abdominal spinal nerves [1].

In recent years a number of important fascial plane blocks have been described blocking the dorsal, lateral and anterior cutaneous nerves of the thorax and abdomen. These new descriptions in blocks are supposed to be an advance in regional anaesthesia due to its simplicity and lack of complications. These include the transversus abdominis plane block, rectus sheath block, quadratus lumborum block, pectoralis nerve block, serratus plane block, retrolaminar block, and now the ESP block [1].

The key advantage common to all of these blocks is that they are technically easier to perform compared with neuraxial, paraverte- 
bral, nerve plexus, and nerve blocks [2].

These plane blocks, on the other hand, have lower risk for serious complications, because the injection is into tissue plane that is distant from potentially problematic structures.

Since its first description by Forero et al. [1] there have been many articles and case reports including an increasingly number of indications for the ESP block: chronic and acute pain treatment [1], rib fractures management in the emergency setting [3,2], treatment abdominal surgical pain [4], hip artroplasty [5] or for analgesic management in breast surgery $[6,7]$; as example of the rapid increase of the number of papers on this matter.

The aim of this narrative review is study the anatomy, mechanism of action and the new indications of this novel technique. It has been reported to be used in both adult and children, however, in this review we will focus on adult anaesthesia papers.

\section{Anatomy}

The ESP block targets the erector spinae plane, which lies in the chest wall between the anterior surface of the cephalo caudal oriented erector spinae muscles and the posterior surface of the spinal transverse processes. LA is deposited in the fascial plane deep to the erector spinae muscle and superficial to the tips of the transverse processes, from where it diffuses to the dorsal and ventral rami of spinal nerves, achieving an extensive multi-dermatomal sensory block of the posterior, lateral, and anterior thoracic wall [1-8].

Anatomically, three muscles trapezius (uppermost), rhomboids major (middle), and erector spinae (lowermost) are identified superficial to the tip of the hyperechoic transverse processes. The erector spinae muscle is not a single muscle, is a structure that forms the paraspinal column, it is a complex composite of three muscles: iliocostalis, longissimus and spinalis, that arise from and insert into various bony components of the vertebral column, for example, from spinous process to spinous process, rib to rib, and transverse process to transverse process [9]. It originates from the sacrum and the lumbar spinous processes, and extends upwards as a gradually tapering column of muscle in the paravertebral groove on either side of the spinous processes, with insertions on the thoracic and cervical vertebrae as high as $\mathrm{C} 2$. This muscular column is encased in a retinaculum (a complex sheet of blended aponeuroses and fasciae) that extends from the sacrum to the skull base. In the lower back this retinaculum is referred to as the thoracolumbar fascia [10]. According to most of authors on this topic, Willard et al.'s paper about the fascia thoracolumbar [10] is required reading for anyone wishing to obtain a deeper understanding of the anatomical basis of this block.

This thoracolumbar fascia, extending from the posterior thorax and abdomen in continuity with the nuchal fascia of the neck, facilitates the spread of LA to multiple thoracic and lumbosacral levels during ESP blocks at lower thoracic levels [11].

According to Hamilton et al, the likely anatomical basis for effective blockade occurs when LA is deposited within the erector sheath [12]. The erector spinae muscles and their associated sheath exhibit a complex three-dimensional anatomy. It is similar to a paired elliptical cylinders one on each side of the vertebral column. Each cylinder is surrounded by a retinacular fascial sheath, separating its contents from the other muscle compartments of the thoracoabdominal cavity. The anterior wall of this fascial sheath is incomplete because the sheath has multiple varied apertures or perforations. Additionally, the sheath is intermittently tethered anteromedially to bony structures along its course, notably the spinous processes and transverse processes of the vertebrae it crosses [12].

The key anatomic factors for ESP block are not so much the nerves but the associated ligaments, fasciae, muscles, and bones, for these latter form the compartment into which the injection is made and the tissue planes within the compartment that determine the spread of de LA [9].

Some authors have hinted at ESP block really being a paravertebral block $[12,9]$ due to there are well-described anatomical gaps in the intertransverse connective tissue that explain how LA can pass from the ESP into the paravertebral space.

Luftig et al reported that there are similarities between ESP block and retrolaminar block mainly in the fact that injection occurs deep to erector spinae muscle in both instances, and that the retrolaminar block probably also works via diffusion of LA into the paravertebral space through the soft tissue gaps between adjacent vertebrae. Nevertheless, the retrolaminar block targets the lamina, and the ESP block targets the transverse process [3]. According to the results of the study in cadavers by Yang et al. both retrolaminar and ESP blocks were consistently associated with the posterior spread of injectate to the back muscles and fascial layers. Regardless of technique, the main route of dye spread 
was through the superior costotransverse ligaments to the affected paravertebral space. The ESP block appears to be slightly more favourable for thoracic spinal nerve blockade than the retrolaminar block in the mid-thoracic region [13]. Additionally, the ESP block produces intercostals spread as well, which may contribute to more extensive analgesia in comparison to retrolaminar block [14].

\section{Mechanism of action}

In the initial report of its description, the authors demonstrated that injection into the fascial plane deep to erector spinae muscle at the level of the T5 transverse process can produce an extensive multi-dermatomal sensory block which was investigated in fresh cadavers, for the likely site of action of ESP block, which being dorsal and ventral rami of thoracic spinal nerves [1].

Cadaveric data showed that LA injected into the tissue plane deep to erector spinae muscle and superficial to the transverse processes and intertransverse connective tissues penetrates anteriorly to anesthetize the spinal nerves [15]. Hamilton and Manickam explained that placing LA in close proximity to the costotransverse foramina, where both the dorsal and ventral rami of the thoracic spinal nerves originate explains its mechanism of action [2].

Additionally, they postulated that both craniocaudal LA spread is facilitated by the thoracolumbar fascia, which extends across the whole of the posterior thorax and abdomen in continuity with the nuchal fascia of the neck superiorly. This provides a logical explanation for the extensive sensory changes and analgesia over the hemithorax after ESP block [2]. As an example, Chin et al reported that an injection of $20 \mathrm{ml}$ into the ESP produces clinical and radiographic evidence of spread that extends at least three vertebral levels cranially and four levels caudally from the site of injection [4].

Anatomical dissection indicates that the likely mechanism of action is diffusion of local anaesthetic anteriorly through the connective tissues and ligaments spanning the adjacent transverse processes and into the vicinity of the spinal nerve roots [4]. A recent study with magnetic resonance imaging demonstrated and confirmed that its mechanism of action is likely linked to the transforaminal and epidural spread, which may be a potential advantage over other thoracic interfascial plane blocks such as pectoralis nerve or serratus anterior and transversus abdominis plane block, because the ESP block provides abdominal visceral analgesia unlike the others [16].
Furthermore, the ESP block has been recently reported to be able to block the sympathetic nerve fibers. [17]. However, the mechanism of sympathetic block is unknown. Ueshima et al [18] experienced with fluoroscopy in patients with a ESP block injecting $15 \mathrm{ml}$ of the radiocontrast agent through the catheter inserted. They investigated the flow and spread of the local anesthetic. The LA solution spread to the thoracic paravertebral space in all cases and more than five intervertebral spaces. Following this, they injected $20 \mathrm{ml}$ of $0.5 \%$ lidocaine through the catheter with the subsequent loss of sensation to pinprick and cold across more than five intervertebral spaces and a reduction in pain score in all patients. They concluded that the local anesthetic solution may spread to the paravertebral space in the ESP block [18].

In fact, the ESP block is considered as a peri-paravertebral regional anesthesia technique [19]. The LA is deposited within the erector sheath compartment, the block will be successful by distribution of the LA not only cranially and caudally along the sheath, but by subsequently gaining access to the paravertebral space via apertures existing in the anterior sheath wall that act as conduits for injected LA. Any injection deeper to the anterior sheath wall does not permit spread of LA beyond one intertransverse space, due to tethering of the sheath to the transverse processes [12]. Hamilton et al reported that the optimal plane for injection may be within the hyperechoic investing sheath, rather than deep to it. In fact, these authors suggested that the ESP block be renamed the erector sheath block in order to highlight the importance of depositing the LA within these anatomical boundaries.

Again and according to Cornish et al ESP block is a "happily accidental" paravertebral block, as we explained above, and that pattern of spread across the aperture in the anterior costotransverse ligament and other anatomical gaps in that connective tissue, explains the main mechanism of action of the ESP block $[9,20]$. However, Chin et al advise that it is an "indirect" paravertebral block: not all of the injected LA will reach the paravertebral space, and thus it may not consistently produce the same degree of sensory block [20].

In contrast, recently Ivanusci et al performed a cadaveric experiment designed to simulate a clinical ESP block documented extensive of dye contrast but they did find no spread of dye anteriorly to the paravertebral space to involve origins of the ventral and dorsal branches of the thoracic spinal nerves. Dor- 
sal ramus involvement was largely posterior to the costotransverse foramen in this experiment. There was potential involvement of the lateral cutaneous branches of the intercostal nerves lateral to the angle of the ribs. Further research is needed to clarify these [21]

On the other hand, there has been consistently reported that the ESP block produced additional spread to intercostals spaces over 5 to 9 levels and was associated with a greater extent of craniocaudal spread along the paraspinal muscles such as Adhikary et al demonstrated with cadaveric observations [22]. Despite of this, Chin et al recommend that it makes more sense to perform an ESP block and inject at a vertebral level congruent to the abdominal surgical incision, rather than relying on adequate spread of the LA. By injecting closer to the neuraxial mid-line, and the paravertebral space, there is also an increased likelihood that the LA will penetrate to the paravertebral space and result in visceral analgesia [23].

Since the first published articles about ESP block at lumbar level [24,5] Kose et al reported that there are several anatomic, sonographic and application differences between ESP block at thoracic or lumbar level [25]. The rigid boundaries of the paravertebral spaces at thoracic level makes that the spread of even small volumes of LA in thoracic ESP block, effects both the ventral and dorsal rami in several levels, leading to multilevel analgesia. However, the paravertebral area does not have such clear boundaries in the lumbar area. Therefore LA is reported to spread partially to the anterior of the paravertebral space at lumbar level. It should be kept in mind that lumbar ESP block from other lumbar levels may lead to different LA spread and therefore varying anesthetic effects [25].

\section{Indications for the ESP block}

\section{Thoracic and Abdominal Chronic and Acute Pain Management}

The first description of this simple interfascial plane block and its successful application in 2 cases of severe thoracic neuropathic pain as well as 2 cases of acute postsurgical pain was published by Forero et al in 2016 [1]. The authors decided to perform a new block because the cranio-caudal extent of the severe thoracic pain made intercostals nerve blocks impractical as well as thoracic epidural or paravertebral injection due to the invasiveness of the techniques and the body habitus of the patients. They decided not to perform a serratus plane block because were concerned that it would not adequately cover the posterior chest wall. With these characteristics they performed an injection within an interfascial plane superficial to the transverse processes/ribs at the site of the patient's primary trigger area for pain, $3 \mathrm{~cm}$ lateral to the T5 spinous process. The result was a successful pain relief with an area of diminished sensation to pinprick extending from T2 to T9, and from $3 \mathrm{~cm}$ laterally to the thoracic spine to the midclavicular line.

Chin et al have demonstrated that the ESP block can indeed provide effective abdominal analgesia following injection at the T7 transverse process [4]. In this report they demonstrated that the ESP block by virtue of its site of action proximal to the origin of the lateral cutaneous branches of the thoracoabdominal wall and the extensive cranio-caudal spread of the injectated LA, can potentially provide sensory blockade of the entire abdominal wall.

On this topic there have been more reports and in 2017 the ESP block has been described in case reports in multiple clinical scenarios including to manage acute postoperative pain in thoracic and abdominal surgeries. Studies have shown that the spread of LA in the paravertebral space in the cephalic and caudal direction can lead to analgesia from C7T2 to L2-3 [4]. For that reason the block was successfully used in acute pain management in pneumothorax surgery (ESP block at T6 level) [26], applied in video-assisted thoracoscopic surgery (VATS) [27], minimally invasive mitral valve surgery via thoracotomy incisions (ESP block at the level of the T7 transverse process) [8], postoperative analgesia after caesarean section (at T9 level) [28], postoperative analgesia in retropubic radical prostatectomy (over the 12th thoracic vertebrae) [29], different laparoscopics abdominal surgeries [19], laparoscopic cholecystectomy in the ambulatory setting [30] and bariatric surgery [17], as examples.

Most part of the literature on this topic are case reports. Recently, Tulgar et al published a randomized controlled clinical trial to evaluate the ESP block for postoperative analgesia in laparoscopic cholecystectomy. In the ESP block group he performed the block at T9 level and they have shown that ESP decreases in postoperative pain intensity in addition to requirement for opioids and other analgesic agents in the first 12 hours after a laparoscopic cholecystectomy [31].

Nevertheless, Takata et al reported in a case series in thoracoscopic lobectomy that ESP block provides tolerable analgesia in thoracoscopic surgery but provides weak dermatomal spread toward the anterior cutaneous branch region, rather than the lateral cutaneous branch region. Thus the author presumes 
that ESP block has the properties of a strong lateral cutaneous branch block that are similar to PECS block, but not to paravertebral or intercostal nerve blocks [32].

\section{Rib fractures}

Useful technique for pain relief in a patient with multiple unilateral rib fractures [2]. Luftig et al reported that the ESP block moves the injection point to be more anatomically aligned with posterior traumatic injury in comparison to serratus anterior plane block [3]. Due to this difference, at that injection point the posterior rami and innervation to the posterior thorax is better targeted. Additionally, injection in this region shows evidence of cephalocaudal and paravertebral LA spread that reaches the origin of the intercostal nerves resulting in dense hemithorax anesthesia $[1,2,33]$. The author suggested that ESP block is highly effective for the acutely injured patient with posterior rib fractures [3].

\section{Breast surgery}

In regard to breast surgery clinicians are seeking simple, safe and effective novel regional anesthesia techniques for postoperative analgesia in this surgery [7]. According to the literature, ESP block seems to cover more surgical areas than other blocks due to its ability to block the anterior cutaneous branches of the intercostal nerves [14]. Gürkan $Y$ et al reported in a randomized controlled study in breast cancer surgery that a single-shot ESP block performed at the T4 thoracic level significantly reduced morphine consumption at the postoperative period [34].

However, Ueshima et al suggested that ESP block alone may not be sufficient to achieve adequate analgesia of anterior branches of T2-T6 and to provide full analgesia necessary for breast cancer surgeries [35]. In fact, the ESP block failed in two case reports and their explanation is based on the cadaveric study by Ivanusic et al, where the authors did not find extension of the dyed contrast to the paravertebral space and dyed only posterior and lateral branched of thoracic nerve [21]. The ESP block may not be able to block anterior branches of intercostal nerve, consequently, further research is necessary to clarify this limitation.

\section{Continuous ESP block}

The ESP also lends itself well to catheter insertion for intermittent boluses or contin- uous perfusion of LA, just as Forero et al described in a case report about the use of a continuous ESP block as rescue analgesia in thoracotomy after epidural failure. The ESP block was performed at $\mathrm{T} 5$ level followed by insertion of a 19-gauge catheter under direct vision $5 \mathrm{~cm}$ beyond the needle tip [15] and the pain relief under a continuous perfusion was satisfactory.

The number of studies reporting the use of continuous ESP block through catheter for postoperative analgesia as part of multimodal anesthesia is increasing. Chung et al reported their experience of a continuous ESP block at the L4 level in a patient with a lower extremity complex regional pain syndrome. In this case the catheterization was maintained for 12 days without any complications and with successful pain management [11].

\section{Cervical level}

Since the erector spinae muscle extends to the cervical spine, the ESP block may be potentially useful in painful conditions of the shoulder girdle. Forero et al performed a series of ESP blocks at the T2/T3 level in a patient with chronic shoulder pain with successful results in pain relief. There was detectable sensory block in the congruent cervico-thoracic dermatomes with no motor block. Computed tomography imaging showed the spread of radiocontrast up to the C3 level in the vicinity of the neural foramina [36].

ESP block application at the T2/3 level for postoperative analgesia in carotid endarterectomy surgery by Ueshima et al is other new indication of the technique in cervical vertebral levels [37].

\section{Lumbar level}

As far as we know, due to the erector spinae muscle anatomy with extension to the lumbosacral level, it is conceivable that LA may spread to lumbosacral levels during ESP block at lower lumbar levels [11]. In fact the ESP block has been described as part of multimodal analgesia after spine surgery of lumbar stenosis or prolapsed lumbar disk [38]. The paraspinal muscles and posterior bony elements of the spine are innervated by the dorsal rami of the spinal nerves. These originate shortly after the spinal nerves exit the vertebral foramina and travel posteriorly through the intertransverse connective tissues and the paraspinal muscles to reach the superficial tissues. In the ESP block LA spreads within the musculofascial plane and acts on the dorsal rami of spinal nerves. Melvin 
et al reported the usefulness of the continuous bilateral ESP block performed at T10/T12 level for perioperative analgesia in lumbrosacral spine surgery in a case series [39].

At lumbar level the ESP block has been also applied as part of multimodal analgesia in a case of transverse process fracture [24]. Tulgar et al also suggested that ESP block performed at L4 level could be as effective as epidural analgesia in total hip arthroplasty [24].

Even though the lumbar approach has been demonstrated reliable and effective, the lumbar ESP block placement can be more challenging due to the increased thickness in the erector spinae muscle and corresponding depth of the intermuscular plane in the lumbar levels, as compared to the thoracic region [40]. For that reason Darling et al find more feasible perform the ESP block at lower thoracic level with the introduction of a directional caudal oriented catheter based on the desired dermatome coverage [40].

\section{Minor surgeries as anesthetic technique}

While generally reported for use in chronic pain and acute postoperative pain, there are also reports of ESP block being used as a surgical anesthesia method in minor surgeries, such as, lipoma excision on the parascapular region under ESP block at T4 [41]. Additionally, Balaban O. et al reported 3 cases of minor surgery at thoracic region under ultrasound guided ESP block [42]. Tulgar et al reported the management of ileostomy closure under ESP block at 8th thoracic vertebral level due to risk of general anesthesia in the patient. High volume and concentration of $0.5 \%$ bupivacaine and $2 \%$ lidocaine was applied with successful result [43].

\section{Other indications}

Its use for other indications, such as pain management in an extensive burn has also recently been reported. Ueshima et al performed an ESP block at T2 vertebral level with insertion of $3 \mathrm{~cm}$ of a catheter into the interfascial space for continuous infusion and the pain symptoms were successfully managed for 2 days [44].

\section{Technique description The right plane block}

This novel interfascial plane block is performed as illustrates the Figure 1. The needle is inserted in a cephalad-to-caudad direction until the tip lay in the interfascial plane between the erector spinae muscles and the transverse process of the targeted vertebral level.

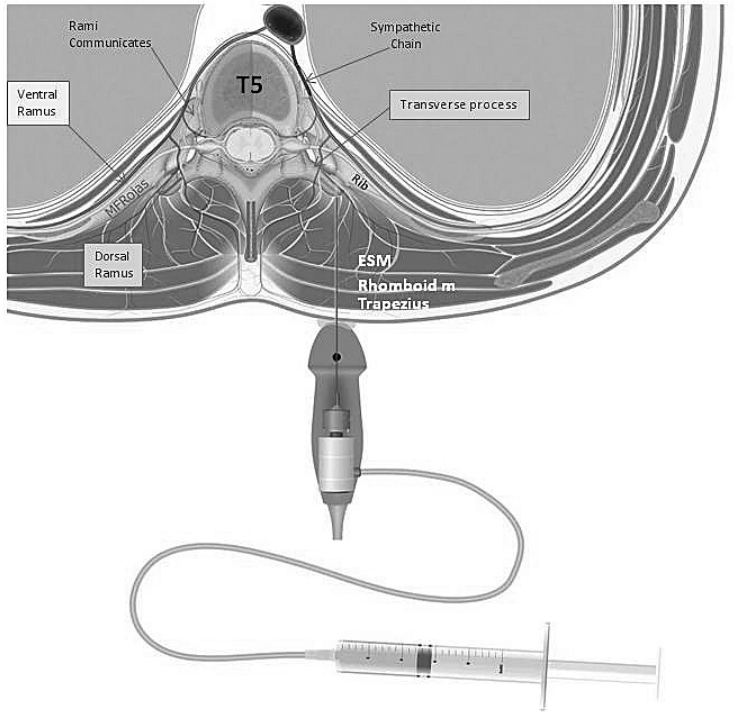

Figure 1: Anatomical demonstration of correct needle placement and injectate spread between the transverse process and erector spinae muscle, with resultant local anesthetic coverage of ventral and dorsal spinal nerve roots. The erector spinae plane (ESP) block is performed by inserting a needle into the fascial plane between the tip of the transverse process and the deep surface of the erector spinae muscle (ESM). (Used with permission from Maria Fernanda Rojas Gomez)

It is necessary to explain that Forero et al firstly described ESP block successfully applied in the interfascial plane between rhomboideus major muscle and erector spinae muscle for thoracic neuropathic pain. This technique failed in the second patient, and subsequent ESP block was performed deep to erector spinae muscle. In their discussion, the authors clearly state that 'the cadaveric findings and our subsequent clinical experience indicate that the optimal plane for injection in the ESP block is deep to the erector spinae muscle rather than superficial to it. [1]. All subsequent studies of ESP block have used this technique [45].

Nevertheless some authors [46] defend that the injection of LA both planes, deep to the erector spinae muscle and also in between the erector spinae muscle and rhomboideus major muscle, had shown comparable analgesic effects in living subjects. In living subjects, there is more dynamic and extensive spread of drug along tissue planes, perhaps following the course of the medial branch of the dorsal rami which allowed the drug to reach ventral rami [46]. On the other hand, there are additional benefits of inserting drug deep to the erector spinae muscle due to the transverse process 
become a convenient sonographic landmark and backstop for needle advancement, contributing to the ease and safety of the block.

In conclusion, the right plane block should be the plane deep to the erector spinae muscle. Furthermore, it is important to perform the injection close to the midline at the tips of the transverse processes, as the costotransverse foramina are located medial to this parasagittal plane (Figure 1). More laterally, the external and internal intercostal muscles may present a significant barrier to local anesthetic spread. The transverse processes also serves as a convenient sonographic landmark and back stop for needle advancement, contributing to the ease and safety of the block [1]. Key bony structures (spinous process, transverse process and rib) can be differentiated by their shapes and relative depths as the transducer is moved laterally from midline.

\section{Patient and ultrasound system position}

According with the description of Luftig et al [3]: expose the posterior thorax by placing the patient prone, in lateral decubitus, or leaning forward in a seated position.

For the prone position, stand at the head of the bed with the ultrasound system on either side of the bed at the level of the patient's thighs.

For the lateral decubitus position (with patient lying on their unaffected side), sit at the side of the bed facing the patient's back with the ultrasound system on the opposite side of the bed (anterior to the patient).

For the seated position, seat the patient on the edge of the bed leaning forward onto aside table in a position similar to the seated lumbar puncture position. Stand behind the patient with the ultrasound system located on the opposite side of the bed anterior to the patient.

For all positions, elevate the bed to a level where the needle, probe, and ultrasound screen can all be viewed in direct line-of-site with minimal head movement.

\section{Technique}

Luftig et al described with detail how to perform the ESP block: at the targeted vertebral level, place a high-frequency linear transducer in cephalocaudal or longitudinal orientation over the midline of the back to identify the vertebral spinous process. Keeping the probe oriented cephalocaudal, slide the probe approximately $3 \mathrm{~cm}$ laterally towards the side to be blocked, identifying the transverse process injection target (Figure 2).

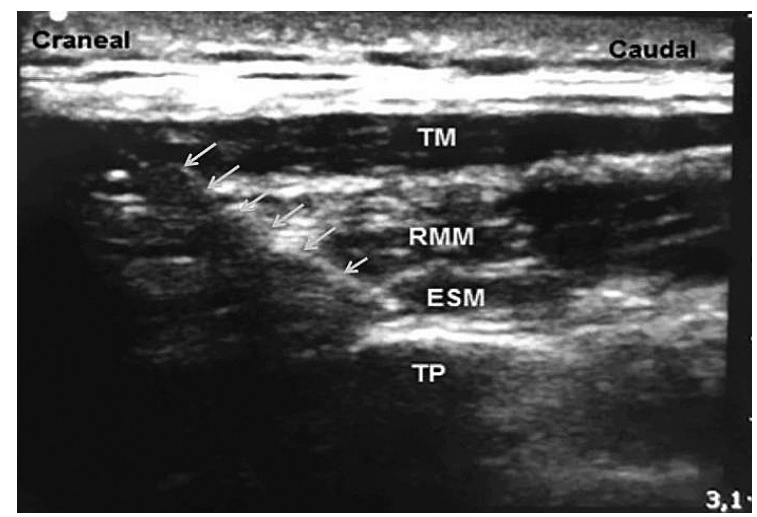

Figure 2: Ultrasound-guided erector spinae plane block. Sonographic demonstration of appropriate needle placement within the erector spinae plane. The needle is inserted in plane through the erector spinae muscle (ESM) to contact the transverse process (TP) (yellow arrows).TM: trapezius muscle, RMM: Rhomboid major muscle

To confirm transverse process identification, slide the probe beyond the target laterally, passing the probe over the costotransverse junction to the rib. The posterior rib adjacent to the costotransverse junction is both lateral and deep to the transverse process. By sliding back and forth over the costotransverse junction, the differentiation between the transverse process and rib will be clear. The transverse process will be more superficial, blunter, wider, while the rib will be deeper, rounder and thinner.

With the transducer fixed over the targeted transverse process, identify a block needle insertion site aligned with the long axis of the ultrasound beam and approximately $1-2 \mathrm{~cm}$ away from the probe (Figure 3 ). Then insert a block needle through the skin and advance at a 30-45-degree angle towards the ultrasound beam. Continue advancing with in-plane ultrasound guidance to the posterior surface of the targeted transverse process. The operator may feel "fascial clicks" corresponding with the fascia of the trapezius, rhomboid (for blocks at T7 and higher), and erector spinae muscles with a final firm end point upon contacting bone.

According to Cornish et al the ESP block should be performed by placing the needle in a cephalad-to-caudal direction onto the superior aspect of the transverse process. This avoids the anterior costotransverse ligament which is not avoided during an insertion of the needle in the opposite direction similar to a paravertebral block approach [9].

Once the needle tip is in the ESP below the erector spinae muscle, it is recommended 


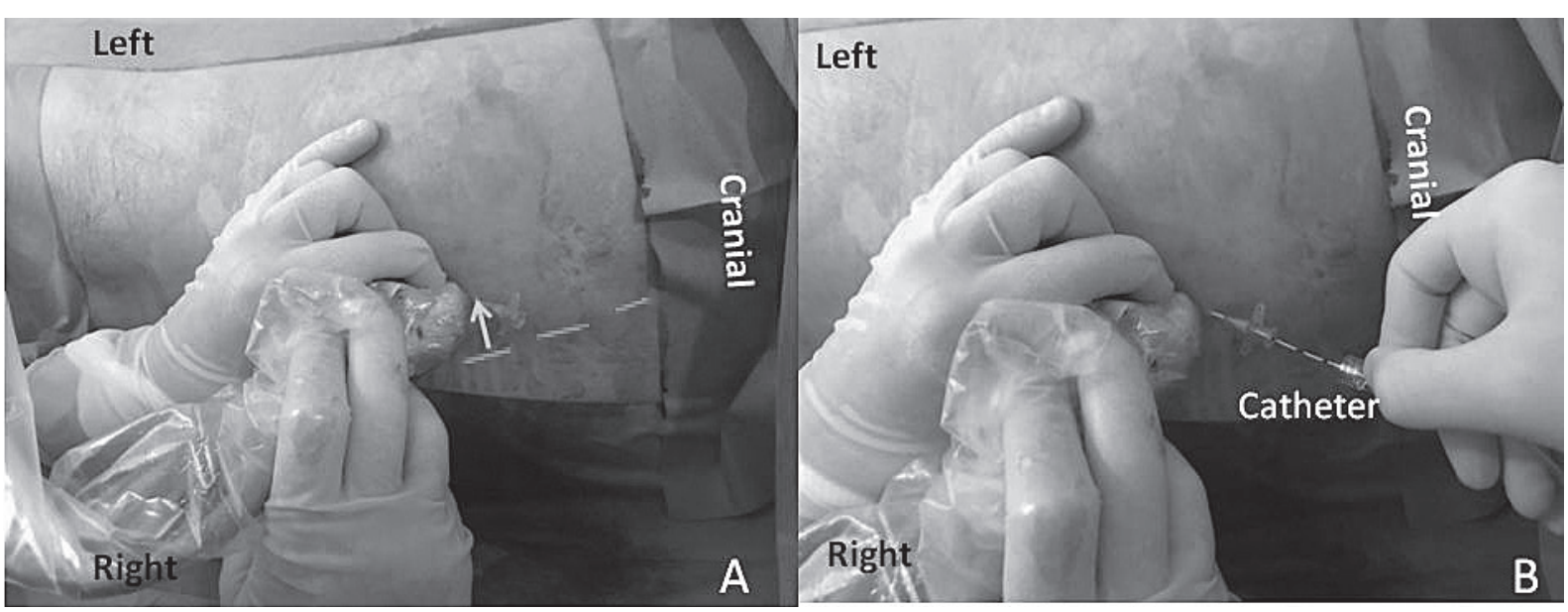

Figure 3: (A) Performance of the erector spinae plane (ESP) block in the right lateral decubitus position. Red lines mark the spinous process and yellow arrow marks $3 \mathrm{~cm}$ laterally where is the target point above the transverse process. (B) The ESP catheter is inserted and secured close to the posterior midline. The needle is oriented in a cephalad-to-caudal direction

alternating aspiration (to confirm lack of inadvertent vascular puncture) with injection of small aliquots of LA. Anechoic fluid should be seen separating the erector spinae muscle from the TP, confirming spread within the ESP. Once satisfied with the needle position, gradually inject LA (3).

Chin et al emphasize that intramuscular injection should be avoided, and that the hyperechoic fascial layer observed between the erector spinae muscle and the transverse processes/intercostal muscles is a complex multi-laminar structure. They therefore usually see LA spreading between two distinct hyperechoic layers rather than purely between the hypoechoic muscle itself and the deeper hyperechoic layer. They believe that it is important to deposit LA deep to the erector spinae muscle to maximize the penetration into the paravertebral space. They recommend that the transverse process should be used as a target for ease and safety of performance, and that the visual end-point that should always be sought is a linear spread of injectate travelling in both a cranial and caudal direction from the point of injection [23].

\section{Doses}

The exact volume and concentration of LA to be used in ESPB is not well established $[47,48]$. From a safety perspective it is recommended large volume of a low LA concentration, however, in some case reports it is used a lower to moderate volume of a high LA concentration [3].

Luftig et al reviewed used doses in ESP block injections documented in the literature [48]. In these case reports, bupivacaine and ropivacaine were the most commonly used
LAs, with injection volumes ranging from 20 $\mathrm{mL}$ to $40 \mathrm{~mL}$, and concentrations ranging from $0.25 \%-0.5 \%$. Taking into consideration the importance of apply the correct dose this author et al created a weight-based LA dose and volume guide for ESP block. That review recommends guidelines limiting bupivacaine doses to $2 \mathrm{mg} /$ $\mathrm{kg}$ (max $175 \mathrm{mg}$ ), and ropivacaine to $3 \mathrm{mg} / \mathrm{kg}$ (max $300 \mathrm{mg}$ ).

It would seem logical that a larger volume would provide for a much more extensive spread in the interfascial plane deep to the erector spinae muscle, even though a higher LA concentration might allow for better diffusion into the paravertebral space [47]. Different concentrations and volumes as well as differing local anesthetics or mixtures remain topics of research for ESPB. Additionally, further studies are necessary to determine the optimal dosing of both single-shot and continuous techniques [31].

On the other hand, due to the lack of evidence in quantity of LA necessary to cover one dermatome after a bolus injection, De Cassai et al [49] performed a review of the medical literature. Indeed, after an injection of LA, the volume needed to cover one dermatome widely varies from $2.5 \mathrm{~mL}[1,26,8,2]$ to $6.6 \mathrm{~mL}[50,7]$, with a median value of $3.4 \mathrm{~mL}$. Furthermore, the maximum number of dermatomes reached by a single bolus in ESP was of 9 dermatomes after a $30 \mathrm{~mL}$ bolus [33]. The author concluded that the value of $3.4 \mathrm{~mL}$ as volume needed to cover one dermatome could be used as a guide to design future studies [49].

\section{Advantages and limitations of the ESP block}




\section{Advantages}

Significant efforts have been made in recent years to identify such alternative regional anaesthetic strategies for analgesia management after surgery, and the recent interest in fascial plane blocks in this clinical setting may signal a paradigm shift by displacing paravertebral or epidural blocks [51]. ESP block is a safer, quicker and less invasive alternative to current standards of analgesia. The time has come to explore easier techniques that are accessible to anaesthetists whose daily practice does not involve wielding an ultrasound probe [51].

ESP has been described as a technically simpler alternative to ultrasound guided paravertebral block with a similar mechanism of action [52,18]. Part of the appeal of the ESP block could be that it is gaining indirect access to the paravertebral space and providing analgesia without the potential for needle-pleura interaction and consequent risk of pneumothorax. There are no structures at risk of needle injury in the immediate vicinity, such as, neuroaxis, pleura, and any major vascular structures. It permits the block to be performed by experienced practitioners in anticoagulated patients with a reasonable safety margin [40]. Similarly some authors believe that injection into a fascial plane and lack of needle proximity to neural structures make it reasonable to perform the ESP block under general anesthesia if necessary [8]. Fortunately, the relatively shallow angle of needle approach allows the broad transverse process to function as a formidable shield for deeper structures. However, complications such as pneumothorax and artery puncture are theoretically possible. Ueshima et al reported a case of pneumothorax after the ESP block in a woman who underwent a left total mastectomy. They recommend to be careful especially in thin patients [53].

Despite its safety, it has been reported an unexpected motor weakness as a side effect of the ESP block carried out at T11 level after cesarean delivery operation. The authors hypothesize that the motor block occurred by a lumbar plexus infiltration of the LA [54].

The sonoanatomy is easily recognizable and the sonographic target is visualized even in the obese patient, making it an attractive option in that patients. The technique also lends itself well to insertion of an indwelling catheter, which can be used to extend the duration of analgesia as needed [1].

The anatomical relation of the erector spinae muscle relative to the vertebra and neu- roforamen enables the clinician to target a wide dermatomal distribution with a single site of injection. It permits to reach effective analgesia and thus, to facilitate opioid sparing, early extubation and effective physiotherapy and mobilization following surgery [8].

\section{Limitations}

Limitations of ESPB should be kept in mind. Positioning to expose the patient's back is required, which can be challenging in the extensively injured patient, such as it is pointed out by Luftig et al [3].

Forero et al reported that, as well as others plane blocks, there are some interindividual variability in the extent of cutaneous block, but this is not unusual in blocks based on local anesthetic spread in tissue planes[1][4].

On the other hand, the fact that LA is deposited distant to the epidural and paravertebral space may, however, be a limitation as well as an advantage. There can be variation in local anesthetic spread, leading in turn to variability in intensity and duration of the block. This can largely be overcome by catheter insertion, which allows redosing by continuous infusion or intermittent boluses [8]. The use of adjuncts such as dexamethasone may help prolong analgesia as well [4].

Other limitation is that the ESP block only provides unilateral thoracic analgesia; bilateral blocks would be required for incisions crossing the midline.

Finally, it should be noted that there is a risk of local anesthetic systemic toxicity with the systemic absorption in ESP block. In order to minimize it, it is preferable to use dilute anesthetic and to add epinephrine in the ESP block when injecting large volumes of LA.

\section{Conclusion}

The ESP block has emerged as a valuable regional anesthesia technique for a range of thoracic, abdominal, and other procedures. The block is gaining popularity as it is easily performed and it has low risk for serious complications. Therefore it seems to have a place as part of a successful multimodal analgesic regimen and comprehensive enhanced recovery post surgery.

Nevertheless, currently the extent of clinical applicability for ESP block has yet to be elucidated, there are limited clinical evidence due to most of the published articles are case reports. Randomized clinical trials are required 
to compare effectiveness of ESP block to other regional analgesia methods. Additionally, further research on confirming the optimal doses and volume of local anesthetic is necessary.

\section{References}

1. Forero M, Adhikary SD, Lopez H, Tsui C, Chin KJ. The Erector Spinae Plane Block: A Novel Analgesic Technique in Thoracic Neuropathic Pain. Reg Anesth Pain Med $2016 ; 41(5): 621-7$.

2. Hamilton DL, Manickam B. Erector spinae plane block for pain relief in rib fractures. $\mathrm{Br}$ J Anaesth 2017;118(3):474-5

3. Luftig J, Mantuani D, Herring AA, Dixon B, Clattenburg E, Nagdev A. Successful emergency pain control for posterior rib fractures with ultrasound-guided erector spinae plane block. Am J Emerg Med;36(8):13916

4. Chin KJ, Adhikary S, Sarwani N, Forero M. The analgesic efficacy of pre-operative bilateral erector spinae plane (ESP) blocks in patients having ventral hernia repair. Anaesthesia 2017;7(4):452-60

5. Tulgar S, Selvi O, Senturk O, Ermis MN, Cubuk R, Ozer Z. Clinical experiences of ultrasound-guided lumbar erector spinae plane block for hip joint and proximal femur surgeries. J Clin Anesth 2018;47:5-6

6. Bonvicini D, Giacomazzi A, Pizzirani E. Use of the ultrasound-guided erector spinae plane block in breast surgery. Minerva Anestesiol 2017;83(10):1111-2

7. Veiga M, Costa D, Brazão I. Erector spinae plane block for radical mastectomy: A new indication? Rev Esp Anestesiol Reanim 2018;65(2):112-5

8. Leyva FM, Mendiola WE, Bonilla AJ, Cubillos J, Moreno DA, Chin KJ. Continuous Erector Spinae Plane (ESP) Block for Postoperative Analgesia after Minimally Invasive Mitral Valve Surgery. J Cardiothorac Vasc Anesth 2017;S1053-0770(17)310054.

9. Cornish PB. Erector Spinae Plane Block: The "Happily Accidental" Paravertebral Block. Reg Anesth Pain Med 2018;43(6):644-645

10. Willard FH, Vleeming A, Schuenke MD, Danneels L, Schleip R. The thoracolumbar fascia: anatomy, function and clinical considerations. J Anat 2012;221(6):507-36

11. Chung K, Kim ED. Continuous erector spinae plane block at the lower lumbar level in a lower extremity complex regional pain syndrome patient. $\mathrm{J}$ Clin Anesth 2018;48:30-1

12. Hamilton DL, Manickam BP. Is the erector spinae plane (ESP) block a sheath block? Anaesthesia 2017;72(7):915-6

13. Yang HM, Choi YJ, Kwon HJ, O J, Cho TH, $\mathrm{Kim} \mathrm{SH}$. Comparison of injectate spread and nerve involvement between retrolaminar and erector spinae plane blocks in the thoracic region: a cadaveric study. Anaesthesia 2018;73(10):1244-1250

14. Adhikary S Das, Bernard S, Lopez $\mathrm{H}$, Chin KJ. Erector Spinae Plane Block Versus Retrolaminar Block: A Magnetic Resonance Imaging and Anatomical Study. Reg Anesth Pain Med 2018; doi:10.1097/ AAP. 0000000000000798

15. Forero $M$, Rajarathinam $M$, Adhikary $S$, Chin KJ. Continuous Erector Spinae Plane Block for Rescue Analgesia in Thoracotomy After Epidural Failure: A Case Report. A A case reports 2017;8(10):254-6

16. Schwartzmann A, Peng $P$, Maciel MA, Forero M. Mechanism of the erector spinae plane block: insights from a magnetic resonance imaging study. Can J Anesth Can d'anesthésie 2018;65(10):1165-1166

17. Chin KJ, Malhas L, Perlas A. The Erector Spinae Plane Block Provides Visceral Abdominal Analgesia in Bariatric Surgery: A Report of 3 Cases. Reg Anesth Pain Med 2017;42(3):372-6

18. Ueshima H, Hiroshi O. Spread of local anesthetic solution in the erector spinae plane block. J Clin Anesth 2018;45:23

19. Tulgar S, Selvi O, Kapakli MS. Erector Spinae Plane Block for Different Laparoscopic Abdominal Surgeries: Case Series. Case Rep Anesthesiol 2018;2018:3947281

20. Chin KJ, Palazzi L, Lapalma J, Forero M, Hernandez MA. Reply to Dr Cornish. Reg Anesth Pain Med 2018;43(6):645-6

21. Ivanusic J, Konishi Y, Barrington MJ. A Cadaveric Study Investigating the Mechanism of Action of Erector Spinae Blockade. Reg Anesth Pain Med 2018;43(6):567-71

22. Adhikary S Das, Bernard S, Lopez H, Chin KJ. Erector Spinae Plane Block Versus Retrolaminar Block. RegAnesth Pain Med 2018; doi:10.1097/AAP.0000000000000798

23. Chin KJ, Adhikary S, Forero M. Is the erector spinae plane (ESP) block a sheath block? A reply. Anaesthesia 2017;72(7):916-7

24. Tulgar S, Senturk O. Ultrasound guided Erector Spinae Plane block at L-4 transverse process level provides effective postoperative analgesia for total hip arthroplas- 
ty. J Clin Anesth 2018;44:68

25. Kose HC, Kose SG, Thomas DT. Lumbar versus thoracic erector spinae plane block: Similar nomenclature, different mechanism of action. J Clin Anesth 2018;48:1

26. Ueshima $\mathrm{H}$, Otake $\mathrm{H}$. Erector spinae plane block provides effective pain management during pneumothorax surgery. J Clin Anesth 2017;40:74

27. Scimia P, Basso Ricci E, Droghetti A, Fusco P. The Ultrasound-Guided Continuous Erector Spinae Plane Block for Postoperative Analgesia in Video-Assisted Thoracoscopic Lobectomy. Reg Anesth Pain Med 2017;42(4):537

28. Yamak Altinpulluk E, García Simón D, Fajardo-Pérez M. Erector spinae plane block for analgesia after lower segment caesarean section: Case report. Rev Esp Anestesiol Reanim 2018;65(5):284-6

29. Tulgar S, Senturk O. Ultrasound guided low thoracic erector spinae plane block for postoperative analgesia in radical retropubic prostatectomy, a new indication. J Clin Anesth 2018;47:4

30. Hannig KE, Jessen C, Soni UK, Børglum J, Bendtsen TF. Erector Spinae Plane Block for Elective Laparoscopic Cholecystectomy in the Ambulatory Surgical Setting. Case Rep Anesthesiol 2018;2018:5492527

31. Tulgar S, Kapakli MS, Senturk O, Selvi O, Serifsoy TE, Ozer Z. Evaluation of ultrasound-guided erector spinae plane block for postoperative analgesia in laparoscopic cholecystectomy: A prospective, randomized, controlled clinical trial. J Clin Anesth 2018;49:101-6

32. Taketa $\mathrm{Y}$, Irisawa $\mathrm{Y}$, Fujitani T. Ultrasound-guided erector spinae plane block elicits sensory loss around the lateral, but not the parasternal, portion of the thorax. J Clin Anesth 2018:47:84-5

33. Forero M, Rajarathinam M, Adhikary S, Chin KJ. Erector spinae plane (ESP) block in the management of post thoracotomy pain syndrome: A case series. Scand J pain 2017;17(1):325-9

34. Gürkan Y, Aksu C, Kuş A, Yörükoğlu UH, Kılıç CT. Ultrasound guided erector spinae plane block reduces postoperative opioid consumption following breast surgery: A randomized controlled study. J Clin Anesth 2018;50:65-8

35. Ueshima $H$, Otake $H$. Limitations of the Erector Spinae Plane (ESP) block for radical mastectomy. J Clin Anesth 2018;51:97

36. Forero $M$, Rajarathinam M, Adhikary $S$ Das, Chin KJ. Erector spinae plane block for the management of chronic shoulder pain: a case report. Can J Anaesth 2018;65(3):288-93

37. Ueshima $H$, Hiroshi $O$. Erector spinae plane block for carotid endarterectomy. J Clin Anesth 2018;48:11

38. Singh S, Chaudhary NK. Bilateral Ultasound Guided Erector Spinae Plane Block for Postoperative Pain Management in Lumbar Spine Surgery: A Case Series. J Neurosurg Anesthesiol 2018;1

39. Melvin JP, Schrot RJ, Chu GM, Chin KJ. Low thoracic erector spinae plane block for perioperative analgesia in lumbosacral spine surgery: a case series. Can J Anaesth 2018;65(9):1057-1065

40. Darling CE, Pun SY, Caruso TJ, Tsui BCH. Successful directional thoracic erector spinae plane block after failed lumbar plexus block in hip joint and proximal femur surgery. J Clin Anesth 2018;49:1-2

41. Ahiskalioglu A, Kocak AO, Doymus O, Sengun E, Celik M, Alici HA. Erector spinae plane block for bilateral lumbar transverse process fracture in emergency department: A new indication. Am J Emerg Med 2018;S0735-6757(18)30554-0

42. Balaban $\mathrm{O}$, Aydin T, Yaman M. Is ultrasound guided erector spinae plane block sufficient for surgical anesthesia in minor surgery at thoracal region? J Clin Anesth 2018;47:7-8

43. Tulgar S, Thomas DT, Deveci U. Erector spinae plane block provides sufficient surgical anesthesia for ileostomy closure in a high-risk patient. J Clin Anesth 2018;48:2-3

44. Ueshima $H$, Otake $H$. Continuous erector spinae plane block for pain management of an extensive burn. Am J Emerg Med 2018; https://doi.org/10.1016/j.ajem.2018.07.014

45. Tulgar S, Balaban O. Local anaesthetic injection point of erector spinae plane block. Indian J Anaesth 2018;62(5):403-4

46. Chaudhary NK, Singh S. The right plane for drug injection in ultrasound-guided erector spinae plane block. Indian J Anaesth 2018;62(5):405

47. Kashani HH, Grocott HP. Clarity needed as to the optimal dose and volume of local anesthetic for erector spinae plane blockade for posterior rib fractures. Am J Emerg Med 2018;36(6):1102-3

48. Josh Luftig P, Mantuani D, Herring AA, Dixon B, Clattenburg E, Nagdev A. The authors reply to the optimal dose and volume of local anesthetic for erector spinae plane blockade for posterior rib fractures. Am J Emerg Med 2018;36(6):1103-4

49. De Cassai A, Tonetti T. Local anesthetic 
spread during erector spinae plane block. J Clin Anesth 2018;48:60-1

50. Ueshima $\mathrm{H}$, Otake $\mathrm{H}$. Clinical experiences of ultrasound-guided erector spinae plane block for thoracic vertebra surgery. J Clin Anesth 2017;38:137

51. El-Boghdadly K, Pawa A. The erector spinae plane block: plane and simple. Anaesthesia 2017;72(4):434-8

52. Fusco P, DI Carlo S, Scimia P, Luciani A, Petrucci E, Marinangeli F. Could the new ultrasound-guided erector spinae plane block be a valid alternative to paravertebral block in chronic chest pain syndromes? Minerva Anestesiol 2017;83(10):1112-3

53. Ueshima $H$. Pneumothorax after the erector spinae plane block. J Clin Anesth 2018;48:12

54. Selvi O, Tulgar S. Ultrasound guided erector spinae plane block as a cause of unintended motor block. Rev Esp Anestesiol Reanim 2018;S0034-9356(18)30110-5 